\title{
QUADRATIC OPERATORS DO NOT GENERATE MAXIMAL LEFT IDEALS OF THE WEYL ALGEBRA
}

\author{
S. C. COUTINHO* \\ Departamento de Ciências da Computacão, Instituto de Matemática, \\ Universidade Federal do Rio de Janeiro, P.O. Box 68530, 21945-970 Rio de Janeiro, RJ, Brazil \\ e-mail:collier@impa.br
}

(Received 2 November, 1998)

\begin{abstract}
We prove that if $n \geq 2$ then $A_{n} d$ cannot be a maximal left ideal of the $n$-th Weyl algebra $A_{n}$ over $\mathbb{C}$ for almost every operator $d$ of degree 2 of $A_{n}$.
\end{abstract}

1991 Mathematics Subject Classification. Primary 16S32.

In Section 5.2 of [1], Bernstein and Lunts claim that an operator of $A_{n}$ of degree 2 (with respect to the Bernstein filtration) cannot generate a maximal left ideal of $A_{n}$. For the sake of simplicity they give a proof under the assumption that the operator of degree 2 has a generic principal symbol. Moreover, their proof is more of a sketch than a complete proof, and it is not really elementary. In this note we give a completely elementary proof of a result that includes the generic result proved by Bernstein and Lunts. For unexplained terminology and for more information on the Weyl algebra see [2].

Throughout this note we will denote by $B_{k}$ the $k$-th component of the Bernstein filtration of the complex Weyl algebra $A_{n}$. Recall that $A_{n}$ is generated over $\mathbb{C}$ by $n$ commuting variables $x_{1}, \ldots, x_{n}$ and their partial differential operators, denoted respectively by $\partial_{1}, \ldots, \partial_{n}$. We will say that an element of $A_{n}$ is a homogeneous operator of degree $r \geq 1$ if it can be written as a linear combination of standard monomials $x^{\alpha} \partial^{\beta}$ for which $|\alpha|+|\beta|=r$. The principal symbol of $d \in A_{n}$ with respect to the Bernstein filtration will be denoted by $\sigma(d)$. Thus, setting $\sigma\left(x_{i}\right)=y_{i}$ and $\sigma\left(\partial_{i}\right)=\xi_{i}$ we have $\sigma\left(x^{\alpha} \partial^{\beta}\right)=y^{\alpha} \xi^{\beta}$. Therefore, by assuming that the monomials of a symbol are always to be written in the form $y^{\alpha} \xi^{\beta}$, we conclude that a homogeneous operator is uniquely determined by its symbol.

Let $V_{1}$ be the complex vector space with basis $x_{1}, \ldots, x_{n}$ and $\partial_{1}, \ldots, \partial_{n}$. One easily checks that

$$
\left[x^{\alpha} \partial^{\beta}, x_{i}\right]=\beta_{i} x^{\alpha} \partial^{\beta-e_{i}} \text { and }\left[x^{\alpha} \partial^{\beta}, \partial_{i}\right]=-\alpha_{i} x^{\alpha-e_{i}} \partial^{\beta},
$$

where $e_{i}$ is the $n$-vector with 1 in the $i$-th entry and zeros elsewhere. Thus a homogeneous operator $P$ of degree 2 induces a vector space endomorphism ad $\operatorname{af}_{d} V_{1}$ by mapping $u \in V_{1}$ to $[P, u]$. Moreover if $P$ has degree 1 , then the same rule determines an element of the dual $V_{1}^{\star}$.

The symbols of operators of degree 2 in $n$ variables are quadratic polynomials in $2 n$ variables and are thus parametrized by $\mathbb{C}^{N}$, where $N=(2 n+1)(n+1)$. Let $\mathcal{O}_{n}$ be

*The work on this paper was partially supported by grants from CNPq and PRONEX(Commutative Algebra and Algebraic Geometry). 
the set of points in $\mathbb{C}^{N}$ that correspond to polynomials $p$ that satisfy both of the following conditions:

(1) $p$ is irreducible;

(2) if $P$ is the homogeneous operator of degree 2 whose symbol is $p$ then $\operatorname{ad}_{P}^{n} \neq 0$.

One immediately checks that $\mathcal{O}_{n}$ is a Zariski open set of $\mathbb{C}^{N}$. Moreover if $n \geq 2$, then $\mathcal{O}_{n}$ is not empty because the symbol of $P=x_{1} \partial_{1}+x_{2} \partial_{2}$ is irreducible, and $\left[P, x_{1}\right]=x_{1}$. In particular, conditions (1) and (2) hold 'generically', in the sense of [1], when $n \geq 2$.

THEOREM. If $d$ is an operator of degree 2 of $A_{n}$ whose symbol corresponds to a point in $\mathcal{O}_{n}$, then $A_{n} d$ is not a maximal left ideal of $A_{n}$.

Proof. We may write $d$ in the form $d=d_{2}+d_{1}+d_{0}$, where $d_{i}$ is a homogeneous operator of degree $i$. Since the vector space endomorphism $\operatorname{ad}_{d_{2}}$ is not nilpotent by condition (2), it follows that it must have a non-zero eigenvalue $\lambda \in \mathbb{C}$. Thus there must exist $0 \neq v \in V_{1}$, an operator of degree 1 , such that $\left[d_{2}, v\right]=\lambda v$. Choose $c \in \mathbb{C}$ such that $\left[d_{1}, v\right]=c \lambda$, and write $u=v+c$. A straightforward computation shows that $[d, u]=\lambda u$. We claim that $A_{n} d+A_{n} u$ is a proper left ideal of $A_{n}$. Since this ideal contains $A_{n} d$ properly, it follows that $A_{n} d$ is not maximal.

Suppose, by contradiction, that $A_{n} d+A_{n} u$ is not a proper ideal of $A_{n}$; in other words $A_{n} d+A_{n} u=A_{n}$. Choose $a, b \in A_{n}$ such that $a d+b u=1$, with $a$ of the smallest possible degree. Note that neither $a$ nor $b$ can be zero. Taking principal symbols we have that

$$
\sigma(a) \sigma(d)+\sigma(b) \sigma(u)=0
$$

Since $\sigma(u)$ has degree 1 and $\sigma(d)$ is irreducible by condition (1), we conclude that there exist $\alpha, a^{\prime}, b^{\prime} \in A_{n}, \alpha \neq 0$, such that $a=-\alpha u+a^{\prime}$ and $b=\alpha d+b^{\prime}$, where $a^{\prime}$ has smaller degree than $a$, and $b^{\prime}$ has smaller degree than $b$. Hence

$$
1=\left(-\alpha u+a^{\prime}\right) d+\left(\alpha d+b^{\prime}\right) u,
$$

so that

$$
1=\alpha[d, u]+\left(a^{\prime} d+b^{\prime} u\right)
$$

Since $[d, u]=\lambda u$ we end up with

$$
1=a^{\prime} d+\left(b^{\prime}+\lambda \alpha\right) u
$$

which contradicts the minimality of the degree of $a$. Therefore $A_{n} d+A_{n} u \neq A_{n}$, and the proof is complete.

\section{REFERENCES}

1. I. N. Bernstein and V. Lunts, On non-holonomic irreducible $D$-modules, Invent. Math. 94 (1988), 223-243.

2. S. C. Coutinho, A primer of algebraic D-modules, London Mathematical Society Student Texts No. 33 (Cambridge University Press, 1995). 